mildly elevated, MRI Brain was normal and neurophysiological testing revealed a diffuse motor neuronopathy. Genetic testing for SMN1 gene was normal. UBA1 sequencing revealed a maternally inherited hemizygous familial variant [c.1681G >A p. (Asp561Asn)], which has not been previously reported.

Results To our knowledge, only six families with SMA2 have been confirmed at a molecular level to date.

Conclusions After ruling out $5 \mathrm{q}$ SMA, $\times$ linked infantile spinal muscular atrophy should be considered in male patients with anterior horn cell disease in the absence of contractures.

\section{P497 REHABILITATION OF CHILDREN WITH SPASTIC AND DYSKINETIC FORMS OF CEREBRAL PALSY THROUGH TRANSCRANIAL EXPOSURE AND BIOLOGICALLY FEEDBACK}

Tatyana Belogorova, Anastasiya Vlasenko, Vanda Mikhnovich, Natalya Dutova, Tatyana Taskaeva, Olga Bugun, Lyubov Rychkova*. Scientific Centre for Family Health and Human Reproduction Problems, Irkutsk, Russian Federation

\subsection{6/archdischild-2019-epa.833}

Movement disorders are determining clinical syndrome of cerebral palsy (CP). The prevalence of $\mathrm{CP}$ in Russia is 2.2-3.3 cases per 1000 newborns; $85-90 \%$ of them are spastic forms. Complex treatment of children with spastic CP includes Botulinum toxin type A as the main component - it reduces spasticity, which optimizes the subsequent rehabilitation. Combination of botulinum therapy with physiotherapy increases the effectiveness of management $\mathrm{CP}$ in children. Transcranial magnetic stimulation was carried out on the 'Amo Atos' device using the add-on device 'OGOLOVYE', according to our own method with a bitemporal arrangement of emitters, with increasing frequency in subsequent procedures from $5 \mathrm{~Hz}$ to $10 \mathrm{~Hz}$, for 10 minutes. Transcranial magnetic stimulation was combined with physical exercise on the device with biofeedback 'MOTOmed gracile' according to an individual scheme after botulinotherapy.

Aim To evaluate the effectiveness of combined use of transcranial magnetic stimulation and physical exercise using the biofeedback device with simultaneous neurometabolic medical therapy, exercise therapy, massage after administration of botulinum toxin type A.

Materials and methods The case-control study included 30 patients 2-14 years old with spastic and dyskinetic CP, comparable by sex, age, degree of motor disorder. The degree of motor disorders was determined by Gross Motor Function Classification System. Evaluation of effectiveness - according to muscle strength scale; tone was estimated by Modified Ashworth Scale; detailed assessment of motor skills according to GMFM-66/88 table, considering the quantitative indicator of motor development. Patients of both groups received botulinum therapy, nicotinic acid, massage, exercise therapy. The main group received physical load on the device 'MOTOmed gracile', transcranial magnetic stimulation; the control group underwent sinusoidal magnetic fields.

Results A 1 point decrease in muscle tone by 10 days was significantly achieved in $92 \%$ of children of the main group and in $75 \%$ of the children in the control group; change in strength was found in $25 \%$ of children in the main and $12 \%$ of the control group; improvement in motor skills was observed in all patients, in the main group by $5.5 \%$, in the control group - by $3 \%$.

Conclusions The study shows high efficacy of botulinum toxin, a good antispastic effect in both groups. Improvement of motor skills and new motor patterns occurs in a fairly short time only with an active rehabilitation program. It confirms the effectiveness of transcranial magnetic therapy and physical activity on the device with biofeedback 'MOTOmed gracile'.

\section{\begin{tabular}{l|l} 
P498 & BOTULINUM TOXIN THERAPY IN PATIENTS WITH
\end{tabular} CEREBRAL PALSY UNDER TWO YEARS}

${ }^{1}$ Margarita Kuznecova*, ${ }^{2}$ Leyla Namazova-Baranova, ${ }^{1}$ Ayaz Mamedyarov, ${ }^{1}$ Lyudmila Kuzenkova, ${ }^{1}$ Aleksey Kurenkov, ${ }^{1}$ Olga Klochkova. ${ }^{1}$ National Medical Research Center for Children's Health, Moscow, Russian Federationl; ${ }^{2}$ Pirogov Russian National Research Medical University, Moscow, Russian Federation

\subsection{6/archdischild-2019-epa.834}

Background and aims The spasticity is a major clinical sign of cerebral palsy (CP). The most effective and safe method of local spasticity decrease is the botulinum toxin A (BTA) therapy (evidence level A). Studying of early BTA injections in complex rehabilitation of children with $\mathrm{CP}$ is relevant due to the detection of wide range of secondary orthopedic problems in patients with early and severe spasticity.

The objective was to determine the most common spasticity patterns for botulinum toxin therapy in patients with cerebral palsy under 2 years and to calculate the BoNT-A mean effective dose for the first injection.

Patients and methods 60 patients (38 boys, 22 girls) aged from 15 up to 23 months (median age 21 months) with spastic forms of CP. Among all patients 20 had tetraparesis, 30 diplegia, 10 - hemiparesis. The prevalence and intensity of muscle hypertonia was estimated in every limb functional segment with the Modified Ashworth scale (MAS). Injections were performed 'off-label' after the written consent of parents or representatives and with the agreement of the local independent ethics committee. Ultrasound control and Onabotulinum Toxin A were used in this study.

Results Spasticity patterns were divided as shown below: forearm pronation in 51 patients $(85 \%)$, equinus foot deformity - $47(78,3 \%)$, hip adduction and internal rotation - 41 $(68,3 \%)$, elbow flexion - 39 (65\%), hip adduction - 33 (55\%), pollex adduction - 21 (35\%), rectus syndrome - 11 $(18,3 \%)$, shoulder adduction and internal rotation - 10 $(16,7 \%)$, elbow extension - $8(13,3 \%)$, in $5 \%$ and less were knee flexion, wrist flexion, fingers flexion. Regardless of early age $57(95 \%)$ patients required multi-level injections. The total dosage for all patients ranged 50-230 U (4,8-23 U/kg). In each injected muscle tone reduced for at least 1 MAS point. Ranges of Onabotulinum Toxin A dosage per muscle are presented in the Poster.

Discussion Definition of the most essential spasticity patterns and their influence on the child movement development is among the key points for the target muscle selection during BTA therapy. Presented dosage ranges allowed us to reach significant decrease of spasticity in all patients. 